\title{
Influence of anthropometric factors on tumour biological characteristics of colorectal cancer in men and women: a cohort study
}

Jenny Brändstedt ${ }^{1 *}$, Sakarias Wangefjord ${ }^{1}$, Signe Borgquist ${ }^{2}$, Björn Nodin ${ }^{1}$, Jakob Eberhard ${ }^{1,2}$, Jonas Manjer ${ }^{3,4}$ and Karin Jirström ${ }^{1}$

\begin{abstract}
Background: Obesity is a well established risk factor of colorectal cancer (CRC), but how body size influences risk of colorectal cancer defined by key molecular alterations remains unclear. In this study, we investigated the relationship between height, weight, body mass index (BMI), waist- and hip circumference, waist-hip ratio (WHR) and risk of CRC according to expression of beta-catenin, cyclin D1, p53 and microsatellite instability status of the tumours in men and women, respectively.

Methods: Immunohistochemical expression of beta-catenin, cyclin D1, p53 and MSI-screening status was assessed in tissue microarrays with tumours from 584 cases of incident CRC in the Malmö Diet and Cancer Study. Six anthropometric factors: height, weight, BMI, waist- and hip circumference, and WHR were categorized by quartiles of baseline measurements and relative risks of CRC according to expression of beta-catenin, cyclin D1, p53 and MSI status were calculated using multivariate Cox regression models.

Results: High height was associated with risk of cyclin D1 positive, and p53 negative CRC in women but not with any investigative molecular subsets of CRC in men. High weight was associated with beta-catenin positive, cyclin D1 positive, p53 negative and microsatellite stable (MSS) tumours in women, and with beta-catenin negative and p53 positive tumours in men. Increased hip circumference was associated with beta-catenin positive, p53 negative and MSS tumours in women and with beta-catenin negative, cyclin D1 positive, p53 positive and MSS tumours in men. In women, waist circumference and WHR were not associated with any molecular subsets of CRC. In men, both high WHR and high waist circumference were associated with beta-catenin positive, cyclin D1 positive and p53 positive tumours. WHR was also associated with p53 negative CRC, and waist circumference with MSS tumours. High BMI was associated with increased risk of beta-catenin positive and MSS CRC in women, and with beta-catenin positive, cyclin D1 positive and p53 positive tumours in men.

Conclusions: Findings from this large prospective cohort study indicate sex-related differences in the relationship between obesity and CRC risk according to key molecular characteristics, and provide further support of an influence of lifestyle factors on different molecular pathways of colorectal carcinogenesis.
\end{abstract}

\footnotetext{
* Correspondence: jenny.brandstedt@med.lu.se

'Department of Clinical Sciences, Division of Pathology, Lund University,

Skåne University Hospital, Lund, Sweden

Full list of author information is available at the end of the article
} 


\section{Introduction}

Colorectal cancer (CRC) is one of the most common forms of human cancer worldwide with approximately 1 million new cases detected every year [1]. Numerous epidemiological studies have examined the relationship between body weight and risk of CRC, most of which have demonstrated a positive association between a high body weight and an increased risk of CRC, particularly in men [2-4]. However, CRC is a largely heterogenous disease in terms of its biological properties and accumulating evidence suggest that aetiological factors influence the carcinogenetic process differentially according to different molecular pathways [5-8].

Colorectal carcinogenesis can be regarded as a complex process with multigene participation, mainly involving at least three distinct pathogenetic pathways: chromosomal instability (CIN), microsatellite instability (MSI) and CpG island methylator phenotype (CIMP) $[9,10]$. The 'suppressor' pathway involves loss of function of the tumour suppressor genes APC (Adenomatosis Polyposis Coli gene), DCC (Deleted in Colorectal Carcinoma gene), p53, and activation of the proto-oncogene $k$-ras. This pathway accounts for approximately $65-70 \%$ of sporadic CRC $[11,12]$ and for cancers associated with familial adenomatous polyposis (FAP), constituting less than $1 \%$ of all CRC [13].

Beta-catenin is a membrane-associated protein with essential functions in the regulation of cellular adhesion and the major mediator of the Wnt-signaling pathway $[14,15]$. Inactivation of kinases in the APC-complex leads to accumulation of cytoplasmic and nuclear beta-catenin, contributing to tumour progression [16-18]. Morikawa et al. have recently shown that BMI is associated with a higher risk of beta-catenin negative-, but not beta-catenin positive colorectal cancer [19]. Cyclin D1 is activated by WNT/betacatenin signalling after mutation of the adenomatous polyposis coli gene (APC) [20]. Cyclin D1 is an important cell-cycle regulating protein and overexpression is seen in about one third of CRC [21]. Although various studies have linked the CCND1 G870A polymorphism to increased CRC risk, the results remain controversial [22,23].

Inactivation of the p53 pathway by p53 mutations is one of the key genetic steps in colorectal carcinogenesis and approximately $40-50 \%$ of tumours in the colon have alterations in the p53 gene [24-26]. The p53 suppressor gene is involved in numerous cellular processes, including induction of apoptosis and cell-cycle arrest, and p53 also plays an important role in cellular energy metabolism [27-29]. It has been shown that reduced nutrient or energy levels induce p53 [30], and given the important role of diet and lifestyle factors to the etiology of CRC, it can be hypothesized that life style factors are associated with p53 mutations. Very few previous studies have however addressed this question. Slattery et al. have shown a positive relationship between western style diet, but not obesity, and p53 mutations [31].
The second pathway is initiated by germline mutations in the mismatch repair (MMR) genes, e.g. $M L H 1, M S H 2$, MSH6, and PMS2, or somatic tumour MLH1 promoter methylation, leading to microsatellite instability (MSI). MSI is detected in approximately $15 \%$ of sporadic CRC, predominantly tumours located in the proximal colon, and in almost all cancers from patients with hereditary non-polyposis colon cancer (HNPCC), accounting for $3-5 \%$ of all CRC [32-34]. Previous data indicate an association between obesity, MSS and risk of CRC [35].

Taken together, while it is well documented that body size influences CRC risk, also with differences regarding sex, location, and tumour stage [36], it remains unclear how this association differs according to molecular tumour phenotype.

The aim of this study was therefore to examine sexrelated differences in the relationship between anthropometric factors and beta-catenin alterations, expression of cyclin D1 and p53, and MSI screening status of incident CRC in a large population based prospective cohort study $(\mathrm{n}=584)$.

\section{Subjects and methods Study group}

Until end of follow-up 31 December 2008, 584 incident cases of CRC had been registered in the prospective, population-based cohort study Malmö Diet and Cancer Study (MDCS) [37]. Between 1991-1996, a total number of 28098 individuals; $11063(39,4 \%)$ men and 17035 $(60,6 \%)$ women, between $44-74$ years where enrolled from a background population of 74 138. All participants completed the baseline examination, which included a questionnaire, anthropometric measurements and a dietary assessment. The questionnaire covered questions on physical activity, use of tobacco and alcohol, heredity, socio-economic factors, education, occupation, previous and current disease and current medication. In addition, blood samples were collected and stored in $-80^{\circ} \mathrm{C}$. Follow up is performed annually by recordlinkage to national registries for cancer and cause of death. Cases were identified from the Swedish Cancer Registry up until 31 December 2007, and from The Southern Swedish Regional Tumour Registry for the period of 1 January to 31 December 2008. All tumours with available slides or paraffin blocks were histopathologically re-evaluated by a senior pathologist $(\mathrm{KJ})$ on haematoxylin and eosin-stained slides. Histopathological, clinical and treatment data were obtained from the clinical and/or pathology records. Information on vital status and cause of death was obtained from the Swedish Cause of Death Registry up until 31 December 2009. Patient and tumour characteristics of the cohort, including specified location of colonic tumours, have been described in detail previously [38-40]. Ethical permissions for the MDCS (Ref. 51/90), and the present 
study (Ref. 530/2008), were obtained from the Ethics Committee at Lund University.

\section{Anthropometric measurements}

At baseline examination, weight, (multiples of $0.1 \mathrm{~kg}$ ) and height (to the nearest $0.005 \mathrm{~m}$ ) were measured by a trained nurse, and body mass index (BMI) was calculated as $\mathrm{kg} / \mathrm{m}^{2}$. Waist circumference was measured at the midpoint between the lower ribs and the iliac crest, and for hip circumference the level of greatest lateral extension was used. These measurements were estimated to the nearest $0.01 \mathrm{~m}$. The waist and hip circumferences of each participant were used to calculate waist-hip ratio (WHR; $\mathrm{cm} / \mathrm{cm}$ ) as an additional measure of fat distribution.

\section{Tissue microarray (TMA) construction and immunohistochemistry}

Tumours with an insufficient amount of material were excluded, and a total number of $557(89.0 \%)$ tumours were suitable for TMA construction. In brief, two $1.0 \mathrm{~mm}$ cores were taken from each tumour and mounted in a new recipient block using a semi-automated arraying device (TMArrayer, Pathology Devices, Westminster, MD, USA). As demonstrated previously, there was no selection bias regarding the distribution of clinicopathological characteristics between the TMA cohort and the full cohort [39].

For immunohistochemical analysis, $4 \mu \mathrm{m}$ TMA-sections were automatically pre-treated using the PT-link system (DAKO, Glostrup, Denmark) and then stained in an Autostainer Plus (DAKO, Glostrup, Denmark). MSI screening status was evaluated as previously described [41]. Immunohistochemical stainings were evaluated as negative when all tumour cells showed loss of nuclear staining. Surrounding stromal cells and tumour infiltrating lymphocytes served as internal controls for each biopsy core. A nuclear reaction of tumour cells was assessed as a positive staining. MSI screening status was defined in accordance with previous studies [41] whereby tumour samples lacking nuclear staining of MLH1, PMS2, MSH2 or MSH6 were considered to have a positive MSI screening status. Hereafter, tumours with a positive MSI screening status are referred to as MSI and tumours with negative MSI screening status are referred to as MSS.

Immunohistochemical staining of beta-catenin was performed and evaluated as previously described [42], whereby membranous staining was denoted as 0 (present) or 1 (absent), cytoplasmic staining intensity as $0-2$ and nuclear staining intensity as $0-2$. In this study, the analyses were limited to nuclear expression of beta-catenin. Cyclin D1 expression was evaluated as previously described [38] and p53 positivity was defined as $>=50 \%$ tumour cells with strong nuclear staining intensity in accordance with previous studies [40]. All immunohistochemical stainings were evaluated by two independent observers ( $\mathrm{SW}$ and $\mathrm{KJ}$ ), who were blinded to clinical and outcome data. Scoring differences were discussed in order to reach consensus.

\section{Statistical methods}

Distribution of established and potential risk factors for CRC was compared between CRC cases and the rest of the study cohort (Table 1). Distribution of cytoplasmic and nuclear beta-catenin expression, expression of p53 and cyclin D1, and MSI-status is also shown in Table 1. Anthropometric measurements were divided into quartiles. Separate quartiles were calculated for men and women [36]. A Cox proportional hazards analysis was used in order to compare risk of CRC between different categories of anthropometric factors according to betacatenin over-expression, p53, and cyclin D1 expression and MSI screening status according to gender and tumour location, i.e. colon vs rectum. This yielded hazard ratios (HR) with a 95\% confidence interval. Follow-up time was defined as time from baseline to diagnosis, death or end of follow-up 31 December 2009. The proportional hazards assumption was confirmed by a log, - log plot [43]. In the multivariate Cox analysis potential confounders were included, i.e. age (years), educational level (not completed elementary school/elementary school (6-8 years)/ "grundskola" (9-10 years)/"studentexamen" (10-12 years)/ one year after "studentexamen"/university degree), smoking habits (yes regularly, yes occasionally, former smoker, never smoker), and alcohol consumption (g/day) (Table 1). A case-to-case analysis examined the heterogeneity between different tumour subgroups regarding their association to anthropometrics using an unconditional logistic regression model. Chi square test was applied for assessment of the distribution of investigative factors according to baseline characteristics. All statistical analyses were conducted using SPSS version 20 and 21 (SPSS Inc., Chicago, IL, USA). Trend was calculated as linear trend over quartiles. A two-tailed p-value less than 0.05 was regarded as statistically significant.

\section{Results}

\section{Distribution of risk factors in cases and rest of cohort}

As shown in Table 1, CRC cases were slightly older ( $p<0.001$ for both men and women), of higher weight ( $\mathrm{p}=0.014$ for men and $\mathrm{p}=0.008$ for women), had a higher BMI ( $\mathrm{p}=<0.001$ for men and $\mathrm{p}=0.001$ for women), a higher waist circumference $(\mathrm{p}<0.001$ for both men and women), and a higher hip circumference $(\mathrm{p}<0.001$ for men and $\mathrm{p}=0.001$ for women) and a higher WHR in men $(p=0.021)$, than the rest of cohort. Among women, cases had a higher level of education $(\mathrm{p}=0.009)$, and had a lower intake of alcohol $(\mathrm{p}=0.002)$ than the rest of cohort. There was a significant association between beta-catenin positive tumours and level of education $(\mathrm{p}=0.019)$, a 
Table 1 Distribution of risk factors in cases and rest of cohort

\begin{tabular}{|c|c|c|c|c|c|c|c|c|c|c|c|c|c|c|c|}
\hline Characteristics & $\begin{array}{l}\text { Rest of } \\
\text { cohort } \\
n=27514\end{array}$ & $\begin{array}{l}\text { CRC } \\
\text { cases } \\
n=584\end{array}$ & $p$ & $\begin{array}{l}\text { beta-catenin }+ \\
n=304 \\
(61.0 \%)\end{array}$ & $\begin{array}{l}\text { beta- catenin - } \\
\mathrm{n}=194 \\
(39.0 \%)\end{array}$ & $p$ & $\begin{array}{l}\text { Cyclin D1+ } \\
n=400 \\
(80.3 \%)\end{array}$ & $\begin{array}{l}\text { Cyclin D1 - } \\
n=98 \\
(19.7 \%)\end{array}$ & $p$ & $\begin{array}{l}\mathrm{p} 53+ \\
\mathrm{n}=241 \\
(48.3 \%)\end{array}$ & $\begin{array}{l}\text { p53- } \\
n=258 \\
(51.7 \%)\end{array}$ & $p$ & $\begin{array}{l}\text { MSI } \\
n=71 \\
(14.6 \%)\end{array}$ & $\begin{array}{l}\text { MSS } \\
n=416 \\
(85.4 \%)\end{array}$ & $p$ \\
\hline Sex & & & $<0.001$ & & & 0.863 & & & 0.226 & & & 0.722 & & & 0.057 \\
\hline Male (\%) & $10783(39.2)$ & $280(47.9)$ & & $145(47.7)$ & $91(46.9)$ & & $185(46.2)$ & $52(53.1)$ & & $112(46.5)$ & $124(48.1)$ & & $\begin{array}{l}26 \\
(36.6)\end{array}$ & $203(48.8)$ & \\
\hline Female (\%) & $16731(60.8)$ & $304(52.1)$ & & $159(52.3)$ & $103(53.1)$ & & $215(53.8)$ & $46(46.9)$ & & $\begin{array}{l}129 \\
(53.5)\end{array}$ & $\begin{array}{l}134 \\
(51.9)\end{array}$ & & $\begin{array}{l}45 \\
(63.4)\end{array}$ & 213 & \\
\hline Age at baseline (years) & 58.0 & 61.8 & & 61.9 & 62.0 & & 62.3 & 60.2 & & 61.5 & 62.3 & & 64.4 & 61.5 & \\
\hline Male & 59.2 & 61.7 & $<0.001$ & 61.5 & 62.1 & 0.430 & 61.9 & 60.5 & 0.204 & 61.2 & 62.0 & 0.452 & 64.6 & 61.2 & 0.029 \\
\hline Female & 57.3 & 62.1 & $<0.001$ & 62.5 & 61.7 & 0.638 & 62.7 & 59.7 & 0.001 & 61.7 & 62.6 & 0.252 & 64.3 & 61.7 & 0.024 \\
\hline Smoking male (\%) & & & 0.104 & & & 0.749 & & & 0.371 & & & 0.199 & & & 0.793 \\
\hline Regularly & $2572(25.5)$ & $53(18.9)$ & & $25(17.2)$ & $17(18.7)$ & & $35(18.9)$ & $8(15.4)$ & & $17(15.2)$ & 24 (19.2) & & $5(19.2)$ & $38(18.5)$ & \\
\hline Occasionally & $520(4.8)$ & $12(4.3)$ & & $5(3.4)$ & $3(3.3)$ & & $8(4.3)$ & 0 & & $5(4.4)$ & $3(2.4)$ & & $1(3.89$ & $7(3.4)$ & \\
\hline Former smoker & $4635(43.0)$ & $145(51.7)$ & & $80(55.2)$ & $44(48.4)$ & & $94(51.4)$ & $31(59.6)$ & & $66(58.9)$ & $60(48.0)$ & & $\begin{array}{l}11 \\
(42.3)\end{array}$ & $107(52.2)$ & \\
\hline Never smoker & $3046(28.3)$ & $70(25.0)$ & & $35(24.1)$ & $27(29.7)$ & & $48(25.9)$ & $13(25.0)$ & & $24(21.4)$ & $38(30.4)$ & & $9(34.6)$ & $53(25.9)$ & \\
\hline Smoking female (\%) & & & 0.652 & & & 0.592 & & & 0.972 & & & 0.009 & & & 0.694 \\
\hline Regularly & $4976(29.8)$ & $71(23.4)$ & & $36(22.6)$ & $25(24.3)$ & & $49(22.8)$ & $11(23.9)$ & & $26(20.2)$ & $35(26.1)$ & & $\begin{array}{l}12 \\
(26.7)\end{array}$ & $47(22.1)$ & \\
\hline Occasionally & $722(4.3)$ & $8(2.6)$ & & $3(1.9)$ & $4(3.9)$ & & $7(3.2)$ & $1(2.2)$ & & $2(1.6)$ & $6(4.5)$ & & $1(2.2)$ & $7(3.3)$ & \\
\hline Former smoke & $4637(27.7)$ & $87(28.6)$ & & $41(25.7)$ & $30(29.1)$ & & $57(26.5)$ & $13(28.3)$ & & $27(20.9)$ & $43(32.1)$ & & $9(20.0)$ & $59(27.7)$ & \\
\hline Never smoker & $7386(44.2)$ & $138(45.4)$ & & 79 (49.7) & $44(42.7)$ & & $102(47.4)$ & $21(45.7)$ & & $74(57.4)$ & $50(37.3)$ & & $\begin{array}{l}23 \\
(51.1)\end{array}$ & $\begin{array}{l}100 \\
(46.9)\end{array}$ & \\
\hline Level of education male (\%) & & & 0.912 & & & 0.727 & & & 0.760 & & & 0.250 & & & 0.451 \\
\hline Not completed & $85(0.8)$ & $2(0.7)$ & & $1(0.7)$ & 0 & & $1(0.5)$ & 0 & & $1(0.9)$ & 0 & & 0 & $1(0.5)$ & \\
\hline $6-8$ years & $4852(45.1)$ & $137(48.9)$ & & $76(52.4)$ & $41(45.1)$ & & $93(50.3)$ & $25(48.1)$ & & $57(50.9)$ & $61(48.8)$ & & $\begin{array}{l}13 \\
(50.0)\end{array}$ & $102(49.8)$ & \\
\hline $9-10$ years & $2113(19.6)$ & $55(19.6)$ & & $28(19.3)$ & $21(23.1)$ & & $35(18.9)$ & $14(26.9)$ & & $24(21.4)$ & $25(20.0)$ & & $4(15.4)$ & $44(21.5)$ & \\
\hline $10-12$ years & $1279(11.9)$ & $29(10.4)$ & & $16(11.0)$ & $10(11.0)$ & & $21(11.3)$ & $4(7.7)$ & & $7(6.3)$ & $18(14.4)$ & & $6(23.1)$ & $20(9.8)$ & \\
\hline 1 year university & $998(9.3)$ & $19(6.7)$ & & $9(6.2)$ & $5(5.5)$ & & $12(6.5)$ & $2(3.8)$ & & $9(8.0)$ & $5(4.0)$ & & $1(3.8)$ & $13(6.3)$ & \\
\hline University degree & $1424(13.2)$ & $38(13.6)$ & & $15(10.3)$ & $14(15.4)$ & & $23(12.4)$ & $7(13.5)$ & & $14(12.5)$ & $16(12.8)$ & & $2(7.7)$ & $25(12.2)$ & \\
\hline
\end{tabular}


Table 1 Distribution of risk factors in cases and rest of cohort (Continued)

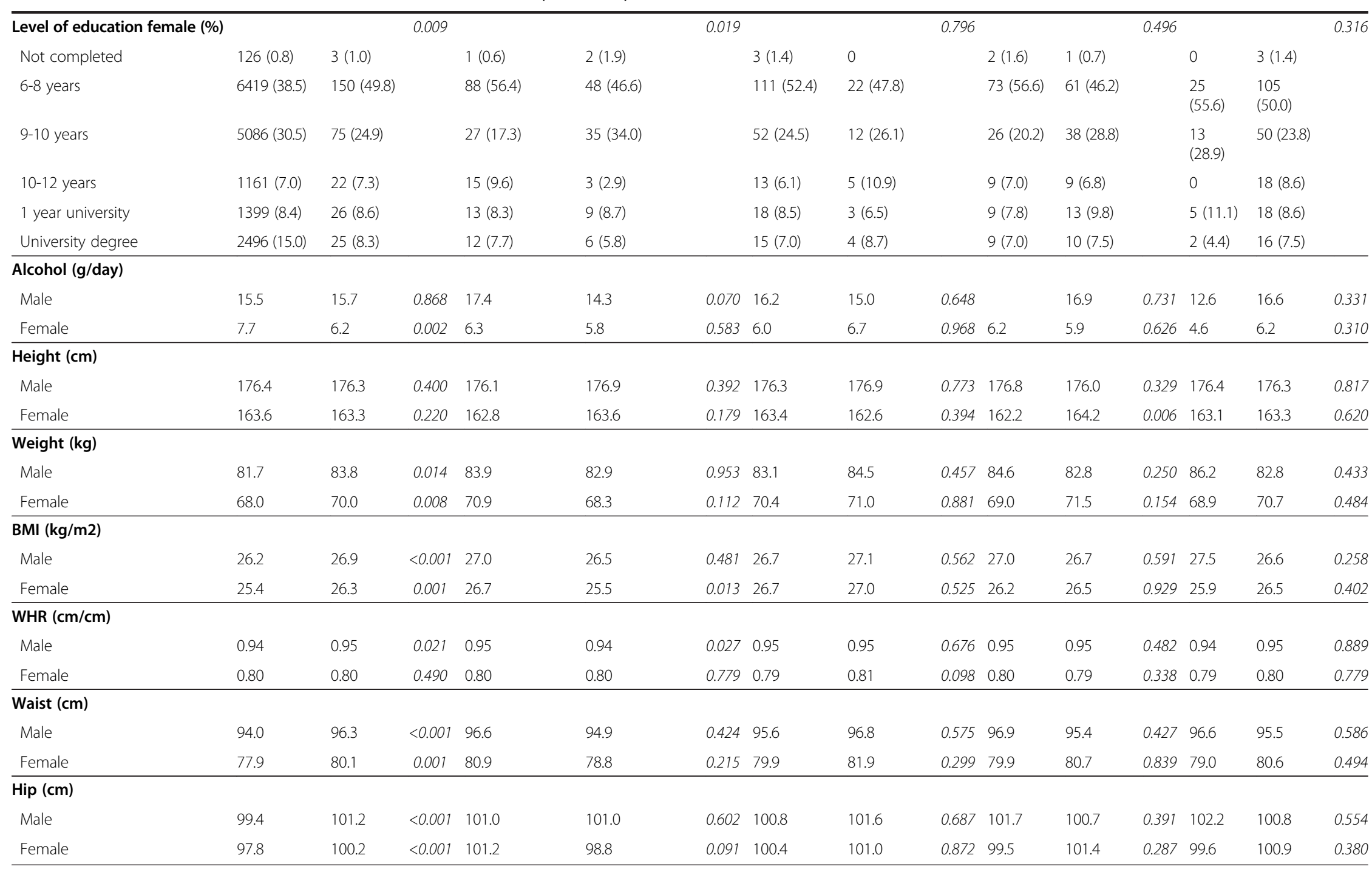


higher BMI ( $\mathrm{p}$ 0.013) in women and with WHR ( $\mathrm{p}=$ 0.027 ) among men. Cyclin D1 positive tumours were associated with higher age $(\mathrm{p}=0.001)$ in women. Furthermore, p53 positive tumours were associated with height $(\mathrm{p}=0.009)$, more frequent among never-smokers in women $(\mathrm{p}=0.009)$, and MSS was associated with higher age in both men $(\mathrm{p}=0.029)$ and in women $(\mathrm{p}=0.024)$.

\section{Hazard ratios of CRC risk defined by different tumour characteristics in women}

Associations of anthropometric factors with tumour biological parameters in women are shown in Table 2 (height, weight, hip) and Table 3 (BMI, WHR, waist). In women, a high height was associated with risk of cyclin D1 positive $\left(p_{\text {trend }}=0.031\right)$, and $\mathrm{p} 53$ negative $\left(p_{\text {trend }}=0.004\right)$ CRC. The risk of p53 negative tumours was highest in the top quartile of height ( $p$ for heterogeneity = 0.013). A high weight was associated with beta-catenin positive $\left(\mathrm{p}_{\text {trend }}=0.010\right)$, cyclinD1 positive $\left(\mathrm{p}_{\text {trend }}=0.019\right), \mathrm{p} 53$ negative $(p=0.004)$ and MSS tumours ( $\left.p_{\text {trend }}=0.008\right)$. Increased hip circumference was associated with betacatenin positive $\left(\mathrm{p}_{\text {trend }}=0.014\right), \mathrm{p} 53$ negative $\left(\mathrm{p}_{\text {trend }}=0.042\right)$ and MSS tumours ( $\mathrm{p}_{\text {trend }}=0.005$ ), but waist circumference and WHR were not associated with risk of any of the molecular subsets of CRC. A high BMI was associated with increased risk of beta-catenin positive $\left(\mathrm{p}_{\text {trend }}=0.004\right)$, but not beta-catenin negative tumours, with the highest risk in the top quartile ( $\mathrm{p}$ for heterogeneity $=0.048$ ). High BMI was also associated with risk of MSS tumours ( $\mathrm{p}_{\text {trend }}=0.009$ ).

\section{Hazard ratios of CRC risk defined by different tumour characteristics in men}

Associations of anthropometric factors with tumour biological parameters in men are shown in Table 4 (height, weight, hip) and Table 5 (BMI, WHR, waist).

High height in men was not associated with increased risk of any of the molecular subsets of CRC, but high weight was associated with beta-catenin negative $\left(\mathrm{p}_{\text {trend }}=0.048\right)$ and $\mathrm{p} 53$ positive $\left(\mathrm{p}_{\text {trend }}=0.026\right)$ CRC. A high hip circumference was associated with beta-catenin negative $\left(\mathrm{p}_{\text {trend }}=0.036\right)$, cyclin D1 positive $\left(\mathrm{p}_{\text {trend }}=0.034\right)$, p 53 positive $\left(p_{\text {trend }}=0.009\right)$ and MSS $\left(p_{\text {trend }}=0.038\right)$ tumours. High BMI was associated with cyclin D1 positive ( $\mathrm{p}_{\text {trend }}=0.019$ ) and $\mathrm{p} 53$ positive $\left(\mathrm{p}_{\text {trend }}=0.023\right.$ ) tumours, and borderline significantly associated with beta-catenin positive CRC ( $\mathrm{p}_{\text {trend }}=0.050$ ). High WHR was associated with beta-catenin positive, but not beta-catenin negative CRC ( $\mathrm{p}_{\text {trend }}=0.001$ ), with the highest risk in the top quartile ( $p$ for heterogeneity $=0.015$ ). A high WHR was also associated with cyclin D1 positive $\left(\mathrm{p}_{\text {trend }}=0.015\right)$, p 53 positive $\left(\mathrm{p}_{\text {trend }}=0.033\right.$ ) and p53 negative tumours $\left(\mathrm{p}_{\text {trend }}=0.048\right)$. High waist circumference was associated with beta-catenin positive $\left(p_{\text {trend }}=0.009\right)$, cyclin D1 positive $\left(\mathrm{p}_{\text {trend }}=0.009\right), \mathrm{p} 53$ positive $\left(\mathrm{p}_{\text {trend }}=0.003\right)$, and MSS ( $\mathrm{p}_{\text {trend }}=0.012$ ) tumours.

\section{Discussion}

In this large prospective cohort study, we present data on associations between anthropometric factors and risk of molecular subsets of CRC, i.e. beta-catenin overexpression, expression of cyclin D1 and p53, and MSI screening status.

Positive MSI screening status has recently been demonstrated to be an independent favourable prognostic factor in the here studied cohort [40]. In the present study, no significant associations were found between any of the anthropometric measurements and risk of MSI tumours. One previous prospective study has investigated the relationship between anthropometric factors and risk of CRC according to MSI status, demonstrating an association of high BMI with MSS tumours but not with MSI tumours [35]. These data are generally in agreement with previous case control studies $[8,44]$. Slattery et al. found that MSI tumours were more common in older people, in women and in the proximal colon, and found a positive relationship between smoking and MSI, and no association between MSI tumours and obesity [8]. In this study, we found significant associations of high weight, BMI and hip circumference with MSS tumours in women. Among men, significant associations were found between increased waist- and hip ratio and hip circumference and MSS tumours. These results are consistent with previous data from Hughes et al. [35], and also generally in agreement with the two previous case control studies from Slattery and Campbell $[8,44]$.

Several anthropometric factors were significantly associated with risk of beta-catenin positive CRC in both sexes; i.e. high weight, BMI and hip circumference in women, and high WHR and waist circumference in men. Differential effects on beta-catenin overexpression, attributable to the top quartiles, were seen for BMI in women and WHR in men. No anthropometric factors were associated with beta-catenin negative tumours in women, whereas in men, high weight and hip circumference were associated with betacatenin negative CRC. Accumulating evidence support a role of $\mathrm{WNT} /$ beta-catenin signalling in adipogenesis, obesity and metabolic disorders [45,46], as well as in carcinogenesis $[14,15]$. Considering the dual roles of betacatenin in both colorectal carcinogenesis and energy metabolism, we investigated potential links between obesity and beta-catenin alterations in CRC. One former study by Morikawa et al. examined the associations of betacatenin expression and obesity with survival from CRC [47], showing an improved cancer-specific survival in obese patients with tumours displaying nuclear betacatenin localization. In non-obese patients, there were no associations between beta-catenin status and survival. 
Table 2 Hazard ratios of CRC risk defined by different tumour characteristics in relation to height, weight and hip circumference in women

\begin{tabular}{|c|c|c|c|c|c|c|c|}
\hline \multirow{2}{*}{$\begin{array}{l}\text { Tumour characteristics } \\
\text { Number of cases }\end{array}$} & \multirow[t]{2}{*}{ Quartiles } & \multicolumn{2}{|c|}{ Height } & \multicolumn{2}{|c|}{ Weight } & \multicolumn{2}{|c|}{ Hip } \\
\hline & & Cases & RR & Cases & $\mathbf{R R}$ & Cases & $\mathbf{R R}$ \\
\hline \multirow[t]{5}{*}{ Positive nuclear beta-catenin } & 1 & 31 & 1.00 & 22 & 1.00 & 19 & 1.00 \\
\hline & 2 & 56 & 1.34(0.86-2.08) & 39 & $1.50(0.89-2.52)$ & 38 & $1.41(0.81-2.46)$ \\
\hline & 3 & 39 & 1.32(0.82-2.13) & 46 & $2.04(1.22-3.41)$ & 37 & $1.28(0.73-2.26)$ \\
\hline & 4 & 32 & $1.17(0.70-1.96)$ & 51 & $1.87(1.12-3.10)$ & 64 & $1.93(1.14-3.26)$ \\
\hline & $p$ trend & & 0.593 & & 0.010 & & 0.014 \\
\hline \multirow[t]{5}{*}{ Negative nuclear beta-catenin } & 1 & 20 & 1.00 & 19 & 1.00 & 21 & 1.00 \\
\hline & 2 & 30 & $1.13(0.64-2.00)$ & 32 & $1.46(0.82-2.57)$ & 21 & $0.72(0.39-1.32)$ \\
\hline & 3 & 26 & $1.33(0.74-2.40)$ & 22 & 1.17(0.63-2.17) & 30 & $1.03(0.58-1.81)$ \\
\hline & 4 & 27 & $1.56(0.86-2.84)$ & 30 & $1.31(0.73-2.33)$ & 31 & $0.88(0.50-1.55)$ \\
\hline & $p$ trend & & 0.113 & & 0.599 & & 0.985 \\
\hline \multirow[t]{5}{*}{ CyclinD1 positive } & 1 & 39 & 1.00 & 35 & 1.00 & 34 & 1.00 \\
\hline & 2 & 70 & $1.36(0.92-2.02)$ & 54 & $1.32(0.86-2.02)$ & 45 & $0.92(0.59-1.44)$ \\
\hline & 3 & 53 & $1.47(0.97-2.23)$ & 56 & $1.57(1.03-2.41)$ & 57 & $1.11(0.72-1.70)$ \\
\hline & 4 & 52 & $1.62(1.05-2.49)$ & 69 & $1.60(1.06-2.42)$ & 78 & $1.28(0.85-1.93)$ \\
\hline & $p$ trend & & 0.031 & & 0.019 & & 0.110 \\
\hline \multirow[t]{5}{*}{ CyclinD1 negative } & 1 & 12 & 1.00 & 6 & 1.00 & 6 & 1.00 \\
\hline & 2 & 14 & $0.80(0.37-1.73)$ & 12 & $1.70(0.63-4.54)$ & 11 & $1.42(0.52-3.85)$ \\
\hline & 3 & 12 & 0.89(0.39-1.99) & 14 & 2.38(0.91-6.22) & 11 & $1.49(0.55-4.07)$ \\
\hline & 4 & 8 & $0.61(0.24-1.53)$ & 14 & $1.96(0.75-5.12)$ & 18 & $2.13(0.83-5.48)$ \\
\hline & $p$ trend & & 0.365 & & 0.155 & & 0.103 \\
\hline \multirow[t]{5}{*}{ p53 positive (>50\%) } & 1 & 30 & 1.00 & 25 & 1.00 & 19 & 1.00 \\
\hline & 2 & 46 & $1.16(0.73-1.84)$ & 32 & $1.07(0.63-1.80)$ & 32 & $1.19(0.67-2.10)$ \\
\hline & 3 & 32 & $1.10(0.67-1.83)$ & 34 & $1.31(0.77-2.20)$ & 34 & $1.19(0.67-2.10)$ \\
\hline & 4 & 21 & $0.78(0.43-1.39)$ & 38 & $1.21(0.72-2.00)$ & 44 & $1.31(0.76-2.28)$ \\
\hline & $p$ trend & & 0.431 & & 0.370 & & 0.364 \\
\hline \multirow[t]{5}{*}{ P53 negative $(<50 \%)$} & 1 & 25 & 1.00 & 16 & 1.00 & 21 & 1.00 \\
\hline & 2 & 40 & $1.39(0.82-2.37)$ & 35 & $1.92(1.06-3.47)$ & 25 & $0.85(0.48-1.53)$ \\
\hline & 3 & 33 & $1.64(0.94-2.84)$ & 37 & $2.36(1.31-4.26)$ & 35 & $1.18(0.68-2.04)$ \\
\hline & 4 & 39 & $2.17(1.25-3.76)^{*}$ & 45 & $2.36(1.33-4.21)$ & 52 & $1.47(0.87-2.47)$ \\
\hline & $p$ trend & & 0.004 & & 0.004 & & 0.042 \\
\hline \multirow[t]{5}{*}{ MSI } & 1 & 11 & 1.00 & 8 & 1.00 & 9 & 1.00 \\
\hline & 2 & 14 & $1.01(0.46-2.23)$ & 12 & $1.35(0.55-3.13)$ & 9 & $0.68(2.271 .71)$ \\
\hline & 3 & 9 & $0.96(0.39-2.33)$ & 11 & $1.49(0.60-3.71)$ & 13 & $0.9(0.40-2.21)$ \\
\hline & 4 & 11 & $1.43(0.61-3.38)$ & 14 & $1.49(0.62-3.59)$ & 14 & $0.81(0.34-1.90)$ \\
\hline & $p$ trend & & 0.477 & & 0.387 & & 0.864 \\
\hline \multirow[t]{5}{*}{ MSS } & 1 & 41 & 1.00 & 30 & 1.00 & 27 & 1.00 \\
\hline & 2 & 67 & $1.20(0.81-1.78)$ & 58 & $1.64(0.05-2.55)$ & 50 & $1.33(0.83-2.13)$ \\
\hline & 3 & 56 & $1.39(0.92-2.09)$ & 55 & $1.79(1.14-2.80)$ & 52 & $1.34(0.84-2.13)$ \\
\hline & 4 & 48 & 1.28(0.83-1.97) & 69 & $1.87(1.21-2.88)$ & 83 & $1.85(1.18-2.88)$ \\
\hline & $p$ trend & & 0.203 & & 0.008 & & 0.005 \\
\hline
\end{tabular}


Table 3 Hazard ratios of CRC risk defined by different tumour characteristics in relation to BMI, WHR and waist and hip circumference in women

\begin{tabular}{|c|c|c|c|c|c|c|c|}
\hline \multirow{2}{*}{$\begin{array}{l}\text { Tumour characteristics } \\
\text { Number of cases }\end{array}$} & \multirow[t]{2}{*}{ Quartiles } & \multicolumn{2}{|r|}{ BMI } & \multicolumn{2}{|c|}{ WHR } & \multicolumn{2}{|c|}{ Waist } \\
\hline & & Cases & RR & Cases & $\mathbf{R R}$ & Cases & $\mathbf{R R}$ \\
\hline \multirow[t]{5}{*}{ Positive nuclear beta-catenin } & 1 & 20 & 1.00 & 43 & 1.00 & 23 & 1.00 \\
\hline & 2 & 40 & $1.73(1.00-2.97)$ & 29 & $0.83(0.52-1.34)$ & 33 & $0.97(0.57-1.67)$ \\
\hline & 3 & 43 & $1.79(1.05-3.05)$ & 40 & $0.92(0.59-1.43)$ & 51 & $1.40(0.85-2.30)$ \\
\hline & 4 & 55 & $2.25(1.33-3.80)^{*}$ & 46 & $1.20(0.79-1.84)$ & 51 & $1.36(0.82-2.25)$ \\
\hline & $p$ trend & & 0.004 & & 0.366 & & 0.097 \\
\hline \multirow[t]{5}{*}{ Negative nuclear beta-catenin } & 1 & 22 & 1.00 & 24 & 1.00 & 16 & 1.00 \\
\hline & 2 & 29 & $1.20(0.69-2.09)$ & 25 & $1.21(0.69-2.12)$ & 25 & $1.16(0.62-2.18)$ \\
\hline & 3 & 28 & $1.06(0.60-1.87)$ & 32 & $1.31(0.77-2.22)$ & 37 & $1.51(0.84-2.73)$ \\
\hline & 4 & 24 & $0.89(0.49-1.61)$ & 22 & $0.97(0.54-1.74)$ & 25 & $1.00(0.53-1.90)$ \\
\hline & $p$ trend & & 0.588 & & 0.952 & & 0.860 \\
\hline \multirow[t]{5}{*}{ CyclinD1 positive } & 1 & 35 & 1.00 & 55 & 1.00 & 31 & 1.00 \\
\hline & 2 & 53 & $1.31(0.85-2.02)$ & 48 & $1.07(0.72-1.58)$ & 49 & $1.09(0.69-1.72)$ \\
\hline & 3 & 61 & $1.42(0.94-2.17)$ & 62 & $1.12(0.77-1.62)$ & 75 & $1.51(0.99-2.31)$ \\
\hline & 4 & 65 & $1.48(0.97-2.25)$ & 49 & $0.98(0.66-1.45)$ & 59 & $1.14(0.73-1.78)$ \\
\hline & $p$ trend & & 0.076 & & 0.997 & & 0.357 \\
\hline \multirow[t]{5}{*}{ CyclinD1 negative } & 1 & 7 & 1.00 & 10 & 1.00 & 8 & 1.00 \\
\hline & 2 & 12 & $1.64(0.64-4.20)$ & 7 & $0.80(0.31-2.11)$ & 7 & $0.69(0.25-1.90)$ \\
\hline & 3 & 9 & $1.19(0.44-3.23)$ & 12 & $1.18(0.51-2.74)$ & 12 & $1.08(0.44-2.66)$ \\
\hline & 4 & 18 & $2.46(1.00-6.02)$ & 17 & $1.92(0.87-4.22)$ & 19 & $1.81(0.78-4.21)$ \\
\hline & $p$ trend & & 0.071 & & 0.066 & & 0.063 \\
\hline \multirow[t]{5}{*}{ p53 positive (>50\%) } & 1 & 19 & 1.00 & 30 & 1.00 & 21 & 1.00 \\
\hline & 2 & 33 & $1.51(0.85-2.67)$ & 27 & $1.10(0.65-1.86)$ & 27 & $0.89(0.50-1.59)$ \\
\hline & 3 & 38 & $1.63(0.93-2.84)$ & 35 & $1.20(0.73-1.96)$ & 40 & $1.20(0.70-2.05)$ \\
\hline & 4 & 39 & $1.65(0.94-2.89)$ & 37 & $1.41(0.87-2.31)$ & 41 & $1.23(0.72-2.10)$ \\
\hline & $p$ trend & & 0.103 & & 0.158 & & 0.248 \\
\hline \multirow[t]{5}{*}{ P53 negative $(<50 \%)$} & 1 & 23 & 1.00 & 35 & 1.00 & 18 & 1.00 \\
\hline & 2 & 33 & $1.29(0.75-2.21)$ & 28 & $0.96(0.58-1.58)$ & 29 & $1.16(0.64-2.09)$ \\
\hline & 3 & 33 & $1.23(0.72-2.11)$ & 39 & 1.08(0.68-1.71) & 49 & $1.77(1.03-3.06)$ \\
\hline & 4 & 44 & $1.61(0.96-2.71)$ & 31 & $0.95(0.58-1.54)$ & 37 & $1.28(0.72-2.27)$ \\
\hline & $p$ trend & & 0.091 & & 0.953 & & 0.236 \\
\hline \multirow[t]{5}{*}{ MSI } & 1 & 10 & 1.00 & 11 & 1.00 & 6 & 1.00 \\
\hline & 2 & 9 & $0.79(0.32-1.95)$ & 10 & $1.08(0.46-2.55)$ & 13 & $1.53(0.58-4.05)$ \\
\hline & 3 & 14 & $1.09(0.48-2.48)$ & 14 & $1.22(0.56-2.70)$ & 16 & $1.59(0.62-4.10)$ \\
\hline & 4 & 12 & $0.91(0.39-2.25)$ & 10 & $0.96(0.41-2.27)$ & 10 & $0.95(0.34-2.65)$ \\
\hline & $p$ trend & & 0.972 & & 0.971 & & 0.765 \\
\hline \multirow[t]{5}{*}{ MSS } & 1 & 31 & 1.00 & 57 & 1.00 & 33 & 1.00 \\
\hline & 2 & 56 & $1.60(1.03-2.50)$ & 44 & $0.94(0.63-1.40)$ & 43 & $0.92(0.58-1.46)$ \\
\hline & 3 & 55 & $1.51(0.97-2.36)$ & 55 & $0.96(0.66-1.40)$ & 69 & $1.37(0.90-2.08)$ \\
\hline & 4 & 70 & $1.90(1.23-2.93)$ & 56 & $1.10(0.76-1.60)$ & 67 & $1.31(0.85-2.00)$ \\
\hline & $p$ trend & & 0.009 & & 0.625 & & 0.065 \\
\hline
\end{tabular}


Table 4 Hazard ratios of CRC risk defined by different tumour characteristics in relation to height, weight and hip circumference in men

\begin{tabular}{|c|c|c|c|c|c|c|c|}
\hline \multirow{2}{*}{$\begin{array}{l}\text { Tumour characteristics } \\
\text { Number of cases }\end{array}$} & \multirow[t]{2}{*}{ Quartiles } & \multicolumn{2}{|c|}{ Height } & \multicolumn{2}{|c|}{ Weight } & \multicolumn{2}{|c|}{ Hip } \\
\hline & & Cases & $\mathbf{R R}$ & Cases & RR & Cases & $\mathbf{R R}$ \\
\hline \multirow[t]{5}{*}{ Positive nuclear beta-catenin } & 1 & 40 & 1.00 & 29 & 1.00 & 26 & 1.00 \\
\hline & 2 & 36 & $0.97(0.61-1.55)$ & 42 & $1.41(0.86-2.29)$ & 33 & $1.28(0.76-2.18)$ \\
\hline & 3 & 33 & $0.71(0.44-1.14)$ & 35 & 1.15(0.69-1.93) & 45 & $1.55(0.93-2.57)$ \\
\hline & 4 & 42 & 1.15(0.73-1.81) & 45 & $1.53(0.94-2.49)$ & 47 & $1.39(0.84-2.30)$ \\
\hline & $p$ trend & & 0.877 & & 0.180 & & 0.182 \\
\hline \multirow[t]{5}{*}{ Negative nuclear beta-catenin } & 1 & 14 & 1.00 & 19 & 1.00 & 17 & 1.00 \\
\hline & 2 & 26 & 2.13(1.09-4.16) & 19 & 1.02(0.53-1.94) & 11 & $0.65(0.30-1.40)$ \\
\hline & 3 & 32 & $1.94(1.00-3.75)$ & 26 & $1.31(0.71-2.43)$ & 33 & $1.87(1.02-3.42)$ \\
\hline & 4 & 27 & $1.90(0.96-3.79)$ & 35 & $1.70(0.94-3.07)$ & 38 & $1.46(0.79-2.70)$ \\
\hline & $p$ trend & & 0.122 & & 0.048 & & 0.036 \\
\hline \multirow[t]{5}{*}{ CyclinD1 positive } & 1 & 45 & 1.00 & 40 & 1.00 & 36 & 1.00 \\
\hline & 2 & 46 & $1.16(0.76-1.77)$ & 49 & $1.23(0.80-1.89)$ & 32 & $0.91(0.56-1.48)$ \\
\hline & 3 & 53 & $1.02(0.67-1.54)$ & 44 & 1.08(0.69-1.68) & 61 & $1.57(1.02-2.41)$ \\
\hline & 4 & 52 & $1.25(0.82-1.90)$ & 63 & $1.57(1.04-2.39)$ & 67 & $1.38(0.90-2.12)$ \\
\hline & $p$ trend & & 0.442 & & 0.058 & & 0.034 \\
\hline \multirow[t]{5}{*}{ CyclinD1 negative } & 1 & 10 & 1.00 & 8 & 1.00 & 7 & 1.00 \\
\hline & 2 & 15 & $1.48(0.66-3.34)$ & 12 & $1.39(0.57-3.42)$ & 12 & $1.65(0.65-4.21)$ \\
\hline & 3 & 13 & $1.00(0.43-2.32)$ & 17 & $1.85(0.79-4.34)$ & 16 & $2.12(0.87-5.18)$ \\
\hline & 4 & 17 & $1.52(0.68-3.43)$ & 18 & 1.78(0.76-4.22) & 20 & $1.90(0.78-4.62)$ \\
\hline & $p$ trend & & 0.513 & & 0.151 & & 0.159 \\
\hline \multirow[t]{5}{*}{ p53 positive (>50\%) } & 1 & 22 & 1.00 & 20 & 1.00 & 15 & 1.00 \\
\hline & 2 & 32 & 1.64(0.93-2.90) & 26 & $1.27(0.70-2.30)$ & 22 & $1.48(0.76-2.90)$ \\
\hline & 3 & 32 & $1.31(0.74-2.31)$ & 33 & $1.42(0.79-2.54)$ & 38 & $2.29(1.23-4.26)$ \\
\hline & 4 & 33 & 1.65(0.93-2.92) & 40 & $1.85(1.06-3.23)$ & 44 & $2.12(1.14-3.92)$ \\
\hline & $p$ trend & & 0.191 & & 0.026 & & 0.009 \\
\hline \multirow[t]{5}{*}{ P53 negative $(<50 \%)$} & 1 & 32 & 1.00 & 27 & 1.00 & 27 & 1.00 \\
\hline & 2 & 30 & $1.02(0.62-1.70)$ & 36 & $1.33(0.79-2.22)$ & 22 & $0.82(0.46-1.46)$ \\
\hline & 3 & 33 & $0.84(0.51-1.39)$ & 27 & $1.06(0.61-1.83)$ & 39 & $1.36(0.82-2.27)$ \\
\hline & 4 & 36 & $1.13(0.68-1.87)$ & 41 & $1.48(0.88-2.48)$ & 43 & $1.15(0.69-1.91)$ \\
\hline & $p$ trend & & 0.845 & & 0.251 & & 0.288 \\
\hline \multirow[t]{5}{*}{ MSI } & 1 & 5 & 1.00 & 4 & 1.00 & 5 & 1.00 \\
\hline & 2 & 9 & $2.07(0.69-6.20)$ & 7 & $1.76(0.51-6.05)$ & 5 & $0.94(0.27-3.26)$ \\
\hline & 3 & 5 & $0.98(0.28-3.42)$ & 7 & $1.91(0.55-6.60)$ & 6 & $1.06(0.32-3.51)$ \\
\hline & 4 & 8 & $1.79(0.55-5.77)$ & 9 & 2.36(0.70-7.96) & 11 & $1.45(0.49-4.32)$ \\
\hline & $p$ trend & & 0.630 & & 0.176 & & 0.431 \\
\hline \multirow[t]{5}{*}{ MSS } & 1 & 48 & 1.00 & 46 & 1.00 & 39 & 1.00 \\
\hline & 2 & 51 & $1.16(0.77-1.74)$ & 51 & $1.09(0.73-1.65)$ & 39 & $1.03(0.65-1.61)$ \\
\hline & 3 & 59 & $1.02(0.69-1.53)$ & 52 & $1.07(0.71-1.62)$ & 64 & $1.54(1.02-2.33)$ \\
\hline & 4 & 58 & $1.25(0.83-1.87)$ & 67 & $1.38(0.93-2.05)$ & 74 & $1.40(0.93-2.11)$ \\
\hline & $p$ trend & & 0.411 & & 0.126 & & 0.038 \\
\hline
\end{tabular}


Table 5 Hazard ratios of CRC risk defined by different tumour characteristics in relation to BMI, WHR and waist circumference in men

\begin{tabular}{|c|c|c|c|c|c|c|c|}
\hline \multirow{2}{*}{$\begin{array}{l}\text { Tumour characteristics } \\
\text { Number of cases }\end{array}$} & \multirow[t]{2}{*}{ Quartiles } & \multicolumn{2}{|c|}{ BMI } & \multicolumn{2}{|c|}{ WHR } & \multicolumn{2}{|c|}{ Waist } \\
\hline & & Cases & HR & Cases & HR & Cases & HR \\
\hline \multirow[t]{5}{*}{ Positive nuclear beta-catenin } & 1 & 33 & 1.00 & 29 & 1.00 & 26 & 1.00 \\
\hline & 2 & 31 & $0.90(0.54-1.48)$ & 28 & $1.21(0.71-2.08)$ & 27 & $0.90(0.52-1.56)$ \\
\hline & 3 & 37 & $0.97(0.60-1.59)$ & 43 & $1.60(0.99-2.59)$ & 47 & $1.36(0.84-2.23)$ \\
\hline & 4 & 50 & $1.52(0.96-2.41)$ & 51 & $2.14(1.34-3.42)^{*}$ & 51 & $1.66(1.02-2.69)$ \\
\hline & $p$ trend & & 0.050 & & 0.001 & & 0.009 \\
\hline \multirow[t]{5}{*}{ Negative nuclear beta-catenin } & 1 & 25 & 1.00 & 30 & 1.00 & 21 & 1.00 \\
\hline & 2 & 15 & $0.57(0.29-1.10)$ & 18 & $0.78(0.43-1.45)$ & 11 & $0.43(0.20-0.91)$ \\
\hline & 3 & 21 & $0.72(0.39-1.33)$ & 26 & $0.98(0.57-1.68)$ & 33 & $1.21(0.69-2.12)$ \\
\hline & 4 & 38 & $1.51(0.86-2.57)$ & 25 & $0.96(0.54-1.69)$ & 34 & $1.27(0.72-2.23)$ \\
\hline & $p$ trend & & 0.074 & & 0.993 & & 0.076 \\
\hline \multirow[t]{5}{*}{ CyclinD1 positive } & 1 & 47 & 1.00 & 45 & 1.00 & 39 & 1.00 \\
\hline & 2 & 33 & $0.69(0.44-1.09)$ & 39 & $1.13(0.72-1.77)$ & 29 & $0.62(0.38-1.02)$ \\
\hline & 3 & 50 & $0.93(0.61-1.42)$ & 54 & $1.33(0.89-2.00)$ & 63 & $1.22(0.80-1.84)$ \\
\hline & 4 & 66 & $1.46(0.99-2.16)$ & 58 & $1.61(1.08-1.42)$ & 65 & $1.40(0.93-2.10)$ \\
\hline & $p$ trend & & 0.019 & & 0.015 & & 0.009 \\
\hline \multirow[t]{5}{*}{ CyclinD1 negative } & 1 & 11 & 1.00 & 14 & 1.00 & 8 & 1.00 \\
\hline & 2 & 12 & $0.95(0.41-2.19)$ & 6 & $0.48(0.17-1.32)$ & 9 & $1.02(0.39-2.64)$ \\
\hline & 3 & 10 & $0.78(0.33-1.86)$ & 17 & $1.28(0.63-2.61)$ & 17 & $1.64(0.70-3.84)$ \\
\hline & 4 & 22 & $1.75(0.83-3.71)$ & 18 & $1.32(0.63-2.72)$ & 21 & $2.01(0.87-4.64)$ \\
\hline & $p$ trend & & 0.146 & & 0.211 & & 0.046 \\
\hline \multirow[t]{5}{*}{ p53 positive (>50\%) } & 1 & 23 & 1.00 & 25 & 1.00 & 16 & 1.00 \\
\hline & 2 & 22 & $0.89(0.49-1.62)$ & 24 & $1.18(0.66-2.12)$ & 21 & $1.05(0.54-2.04)$ \\
\hline & 3 & 32 & $1.17(0.67-2.04)$ & 33 & $1.43(0.84-2.44)$ & 41 & $1.85(1.02-3.33)$ \\
\hline & 4 & 42 & $1.69(0.99-2.88)$ & 37 & $1.72(1.02-2.91)$ & 41 & $2.05(1.14-3.68)$ \\
\hline & $p$ trend & & 0.023 & & 0.033 & & 0.003 \\
\hline \multirow[t]{5}{*}{ P53 negative (<50\%) } & 1 & 34 & 1.00 & 32 & 1.00 & 30 & 1.00 \\
\hline & 2 & 23 & $0.65(0.38-1.13)$ & 22 & $0.91(0.52-1.59)$ & 16 & $0.48(0.26-0.89)$ \\
\hline & 3 & 28 & $0.73(0.43-1.24)$ & 38 & 1.33(0.82-2.15) & 40 & $1.05(0.64-1.71)$ \\
\hline & 4 & 46 & $1.44(0.90-2.30)$ & 39 & $1.52(0.93-2.47)$ & 45 & $1.25(0.76-2.03)$ \\
\hline & $p$ trend & & 0.084 & & 0.048 & & 0.070 \\
\hline \multirow[t]{5}{*}{ MSI } & 1 & 5 & 1.00 & 6 & 1.00 & 5 & 1.00 \\
\hline & 2 & 5 & $0.98(0.28-3.42)$ & 5 & $1.27(0.39-4.18)$ & 4 & $0.73(0.20-2.72)$ \\
\hline & 3 & 6 & $0.95(0.27-3.34)$ & 9 & $1.82(0.64-5.15)$ & 9 & $1.58(0.53-4.75)$ \\
\hline & 4 & 11 & $2.47(0.84-7.26)$ & 7 & $1.52(0.48-4.80)$ & 9 & $1.50(0.49-4.65)$ \\
\hline & $p$ trend & & 0.082 & & 0.344 & & 0.272 \\
\hline \multirow[t]{5}{*}{ MSS } & 1 & 53 & 1.00 & 55 & 1.00 & 43 & 1.00 \\
\hline & 2 & 40 & $0.72(0.47-1.10)$ & 40 & $0.90(0.59-1.39)$ & 34 & $0.67(0.42-1.06)$ \\
\hline & 3 & 50 & $0.84(0.56-1.25)$ & 58 & $1.14(0.78-1.67)$ & 67 & $1.16(0.78-1.73)$ \\
\hline & 4 & 73 & $1.37(0.95-1.99)$ & 63 & 1.36(0.93-1.98) & 72 & $1.39(0.94-2.05)$ \\
\hline & $p$ trend & & 0.053 & & 0.071 & & 0.012 \\
\hline
\end{tabular}


Furthermore, Morikawa et al. have recently presented data on the relationship between obesity, measured as BMI, and risk of $\mathrm{CRC}$ according to beta-catenin status, whereby the results demonstrate that obesity and physical inactivity are associated with a higher risk of betacatenin negative but not of betacatenin positive CRC [19]. Of note, in the MDCS, beta-catenin overexpression has been demonstrated to be significantly associated with favourable clinicopathological factors and a prolonged survival [40].

As regards cyclin D1 expression, the results from the present study demonstrate a significant association between a high height and weight and risk of cyclin D1 positive tumours in women. In men, significant associations were seen between high BMI, WHR, waist and hip circumference and cyclin D1 positive tumours. Notably, in order to avoid too small subgroup analyses, a dichotomized variable of negative vs positive cyclin D1 expression was used, since this cut off takes both nuclear fraction and intensity into account and has previously shown to have the strongest impact on survival [38]. We are not aware of any previous studies on the influence of anthropometric factors on CRC risk according to cyclin D1 expression. Although various studies have linked the CCND1 G870A polymorphism to increased risk of $\mathrm{CRC}$, the findings remain controversial $[22,23]$. The prognostic role of cyclin D1 has been investigated in several studies, however with inconsistent results [48-52]. In a previous study, expression of cyclin D1 was found to be associated with a significantly prolonged survival from CRC in men but not in women in the MDCS [38].

Lastly, the results from the present study demonstrate a positive relationship of all investigated anthropometric factors except height and weight, with p53 positive tumours in men, whereas in women, no associations were found between any anthropometric factors and p53 positive CRC. In contrast, high height, in particular the top quartile, weight and hip circumference were associated with p53 negative tumours in women. As a cautionary remark, the correlation between p53 gene mutations and p53 positivity by immunohistochemistry is not entirely concordant, and these analyses may therefore include some false positive and negative cases [53]. Previous studies on anthropometric factors and risk of CRC according to p53 expression are sparse, and with inconclusive results. Zhang et al. reported a possible association between p53 overexpression and obesity [54], and Slattery et al. have shown a positive relationship between western style diet and p53 mutations, but not between obesity and p53 mutations [31].

Taken together, while it is well documented that body size influences CRC risk, also with differences regarding sex, location, and tumour stage, the exact biologic mechanisms underlying the association between obesity and increased risk of CRC are not fully understood. A large number of studies have shown an increased risk of CRC in men, but not in women, and the complex interplay between hormonal factors and tumour biology underlying these sex differences remains to be further elucidated. Further, our results validate previous findings demonstrating significant associations of obesity and risk of microsatellite unstable, but not microsatellite stable, colorectal cancer in both sexes.

Certain methodological aspects need further attention. The validity of the anthropometric measurements is one aspect, as there may be a potential inter-observer variation. Recommendations for the nurses performing baseline examinations described how participants should be dressed, in which position the participants should be examined, and location for the estimation of waist- and hip measurements. We therefore consider the risk of misclassification of anthropometric measurements to be low. In contrast, most previous studies have used selfreported anthropometric measures.

It is also possible that participation in the MDCS was associated with body constitution, which may have lead to potential selection bias. In a previous paper, Manjer et al. compared BMI in the MDCS population in relation to the background population, and found an equal distribution of overweight/obesity [25]. Another aspect is the validity of collected data. As anthropometric data was assessed only at baseline, it is possible that some individuals have gained and some have lost weight. Such a misclassification is likely to lead to an attenuation of risks and, if anything, observed risks may be underestimated.

\section{Conclusions}

The results from this large prospective cohort study demonstrate that obesity, measured by several anthropometric factors, is differently associated with beta-catenin alterations, expression of cyclin D1 and p53, and MSI screening status of colorectal tumours in men and women. While not allowing for any firm conclusions to be drawn, these findings further support that the influence of lifestyle factors on various pathways of colorectal carcinogenesis differs between sexes. Further study on this topic is encouraged in order to enable development of novel strategies for screening and prevention of colorectal cancer.

\section{Competing interests}

The authors declare that they have no competing interests.

\section{Authors' contributions}

JB performed the statistical analyses and drafted the manuscript. SW collected clinical data and evaluated the immunohistochemical stainings, SB assisted with the statistical analyses and helped draft the manuscript. BN constructed the TMAs and carried out the IHC stainings. JM and JE assisted with the data collection and helped draft the manuscript. KJ conceived of the study, carried out the histopathological re-evaluation, evaluated the immunohistochemical stainings, and helped draft the manuscript. All authors read and approved the final manuscript. 


\section{Acknowledgements}

This study was supported by grants from the Knut and Alice Wallenberg Foundation, the Swedish Cancer Society, the Gunnar Nilsson Cancer Foundation, Region Skåne and the Research Funds of Skåne University Hospital.

\section{Author details}

${ }^{1}$ Department of Clinical Sciences, Division of Pathology, Lund University, Skåne University Hospital, Lund, Sweden. 'Department of Clinical Sciences, Division of Oncology, Lund University, Skåne University Hospital, Lund Sweden. ${ }^{3}$ Department of Clinical Sciences, Division of Surgery, Lund University, Skåne University Hospital, Malmö, Sweden. ${ }^{4}$ The Malmö Diet and Cancer Study, Lund University, Malmö, Sweden.

Received: 27 February 2013 Accepted: 12 November 2013 Published: 21 November 2013

\section{References}

1. Jemal A, Bray F, Center MM, Ferlay J, Ward E, Forman D: Global cancer statistics. CA Cancer J Clin 2011, 61(2):69-90.

2. Pischon $\mathrm{T}$, Lahmann $\mathrm{PH}$, Boeing $\mathrm{H}$, Friedenreich $\mathrm{C}$, Norat $\mathrm{T}$, Tjonneland $\mathrm{A}$, Halkjaer J, Overvad K, Clavel-Chapelon F, Boutron-Ruault MC, et al: Body size and risk of colon and rectal cancer in the european prospective investigation into cancer and nutrition (EPIC). J Natl Cancer Inst 2006, 98(13):920-931.

3. Renehan AG, Tyson M, Egger M, Heller RF, Zwahlen M: Body-mass index and incidence of cancer: a systematic review and meta-analysis of prospective observational studies. Lancet 2008, 371(9612):569-578.

4. Larsson SC, Wolk A: Obesity and colon and rectal cancer risk: a meta-analysis of prospective studies. Am J Clin Nutr 2007, 86(3):556-565.

5. Ogino S, Shima K, Nosho K, Irahara N, Baba Y, Wolpin BM, Giovannucci EL, Meyerhardt JA, Fuchs CS: A cohort study of p27 localization in colon cancer, body mass index, and patient survival. Cancer Epidemiol Biomarkers Prev 2009, 18(6):1849-1858

6. Limsui D, Vierkant RA, Tillmans LS, Wang AH, Weisenberger DJ, Laird PW, Lynch CF, Anderson KE, French AJ, Haile RW, et al: Cigarette smoking and colorectal cancer risk by molecularly defined subtypes. J Natl Cancer Inst 2010, 102(14):1012-1022

7. Satia JA, Keku T, Galanko JA, Martin C, Doctolero RT, Tajima A, Sandler RS, Carethers JM: Diet, lifestyle, and genomic instability in the North Carolina colon cancer study. Cancer Epidemiol Biomarkers Prev 2005, 14(2):429-436.

8. Slattery ML, Curtin K, Anderson K, Ma KN, Ballard L, Edwards S, Schaffer D, Potter J, Leppert M, Samowitz WS: Associations between cigarette smoking, lifestyle factors, and microsatellite instability in colon tumors. J Natl Cancer Inst 2000, 92(22):1831-1836.

9. Armaghany T, Wilson JD, Chu Q, Mills G: Genetic alterations in colorectal cancer. Gastrointest Cancer Res 2012, 5(1):19-27.

10. Pancione M, Remo A, Colantuoni V: Genetic and epigenetic events generate multiple pathways in colorectal cancer progression. Patholog Res Int 2012, 2012:509348.

11. Pino MS, Chung DC: The chromosomal instability pathway in colon cancer. Gastroenterology 2010, 138(6):2059-2072.

12. Al-Sohaily S, Biankin A, Leong R, Kohonen-Corish M, Warusavitarne J: Molecular pathways in colorectal cancer. J Gastroenterol Hepatol 2012, 27(9):1423-1431.

13. Half E, Bercovich D, Rozen P: Familial adenomatous polyposis. Orphanet $J$ Rare Dis 2009, 4:22

14. Kikuchi A: Regulation of beta-catenin signaling in the Wnt pathway. Biochem Biophys Res Commun 2000, 268(2):243-248.

15. Lugli A, Zlobec I, Minoo P, Baker K, Tornillo L, Terracciano L, Jass JR: Prognostic significance of the wnt signalling pathway molecules APC, beta-catenin and E-cadherin in colorectal cancer: a tissue microarray-based analysis. Histopathology 2007, 50(4):453-464.

16. Brabletz $T$, Jung A, Kirchner T: Beta-catenin and the morphogenesis of colorectal cancer. Virchows Arch 2002, 441(1):1-11.

17. Clevers H: Wnt breakers in colon cancer. Cancer Cell 2004, 5(1):5-6.

18. Klaus A, Birchmeier W: Wnt signalling and its impact on development and cancer. Nat Rev Cancer 2008, 8(5):387-398.

19. Morikawa $T$, Kuchiba A, Lochhead P, Nishihara R, Yamauchi M, Imamura $Y$, Liao X, Qian ZR, Ng K, Chan AT, et al: Prospective analysis of body mass index, physical activity, and colorectal cancer risk associated with betacatenin (CTNNB1) status. Cancer Res 2013, 73(5):1600-1610.

20. Tetsu O, McCormick F: Beta-catenin regulates expression of cyclin D1 in colon carcinoma cells. Nature 1999, 398(6726):422-426.

21. Arber N, Hibshoosh H, Moss SF, Sutter T, Zhang Y, Begg M, Wang S, Weinstein IB, Holt PR: Increased expression of cyclin D1 is an early event in multistage colorectal carcinogenesis. Gastroenterology 1996, 110(3):669-674.

22. Yang J, Zhang G, Chen J: CCND1 G870A polymorphism is associated with increased risk of colorectal cancer, especially for sporadic colorectal cancer and in Caucasians: a meta-analysis. Clin Res Hepatol Gastroenterol 2012, 36(2):169-177.

23. Zhang LQ, Wang J, Shang JQ, Bai JL, Liu FY, Guan X, Zhou JN: Cyclin D1 G870A polymorphism and colorectal cancer susceptibility: a meta-analysis of 20 populations. Int J Colorectal Dis 2011, 26(10):1249-1255.

24. Baker SJ, Fearon ER, Nigro JM, Hamilton SR, Preisinger AC, Jessup JM, VanTuinen $\mathrm{P}$, Ledbetter DH, Barker DF, Nakamura Y, et al: Chromosome 17 deletions and p53 gene mutations in colorectal carcinomas. Science 1989, 244:217-221.

25. Baker SJ, Markowitz S, Fearon ER, Willson JK, Vogelstein B: Suppression of human colorectal carcinoma cell growth by wild-type p53. Science 1990 249(4971):912-915.

26. Markowitz SD, Bertagnolli MM: Molecular origins of cancer: molecular basis of colorectal cancer. N Engl J Med 2009, 361(25):2449-2460.

27. Vousden KH, Ryan KM: p53 and metabolism. Nat Rev Cancer 2009, 9(10):691-700.

28. Levine AJ, Puzio-Kuter AM: The control of the metabolic switch in cancers by oncogenes and tumor suppressor genes. Science 2010, 330(6009):1340-1344.

29. Cairns RA, Harris IS, Mak TW: Regulation of cancer cell metabolism. Nat Rev Cancer 2011, 11(2):85-95.

30. Jones RG, Plas DR, Kubek S, Buzzai M, Mu J, Xu Y, Birnbaum MJ, Thompson CB: AMP-activated protein kinase induces a p53-dependent metabolic checkpoint. Mol Cell 2005, 18(3):283-293.

31. Slattery ML, Curtin K, Ma K, Edwards S, Schaffer D, Anderson K, Samowitz W: Diet activity, and lifestyle associations with p53 mutations in colon tumors. Cancer Epidemiol Biomarkers Prev 2002, 11(6):541-548.

32. Samowitz WS, Slattery ML, Kerber RA: Microsatellite instability in human colonic cancer is not a useful clinical indicator of familial colorectal cancer. Gastroenterology 1995, 109(6):1765-1771.

33. Liu B, Nicolaides NC, Markowitz S, Willson JK, Parsons RE, Jen J, Papadopolous N, Peltomaki P, de la Chapelle A, Hamilton SR, et al: Mismatch repair gene defects in sporadic colorectal cancers with microsatellite instability. Nat Genet 1995, 9(1):48-55.

34. Aaltonen LA, Peltomaki P, Mecklin JP, Jarvinen H, Jass JR, Green JS, Lynch HT, Watson $P$, Tallqvist G, Juhola M, et al: Replication errors in benign and malignant tumors from hereditary nonpolyposis colorectal cancer patients. Cancer Res 1994, 54(7):1645-1648.

35. Hughes LA, Williamson EJ, van Engeland M, Jenkins MA, Giles GG, Hopper JL, Southey MC, Young JP, Buchanan DD, Walsh MD, et al: Body size and risk for colorectal cancers showing BRAF mutations or microsatellite instability: a pooled analysis. Int J Epidemio/ 2012, 41(4):1060-1072.

36. Brandstedt J, Wangefjord S, Nodin B, Gaber A, Manjer J, Jirstrom K: Gender, anthropometric factors and risk of colorectal cancer with particular reference to tumour location and TNM stage: a cohort study. Bio/ Sex Differ 2012, 3(1):23.

37. Berglund G, Elmstahl S, Janzon L, Larsson SA: The malmo diet and cancer study. Design and feasibility. J Intern Med 1993, 233(1):45-51.

38. Wangefjord S, Manjer J, Gaber A, Nodin B, Eberhard J, Jirstrom K: Cyclin D1 expression in colorectal cancer is a favorable prognostic factor in men but not in women in a prospective, population-based cohort study. Biology of sex differences 2011, 2:10.

39. Larsson A, Johansson ME, Wangefjord S, Gaber A, Nodin B, Kucharzewska P, Welinder C, Belting M, Eberhard J, Johnsson A, et al: Overexpression of podocalyxin-like protein is an independent factor of poor prognosis in colorectal cancer. Br J Cancer 2011, 105(5):666-672.

40. Wangefjord S, Brandstedt J, Lindquist KE, Nodin B, Jirstrom K, Eberhard J: Associations of beta-catenin alterations and MSI screening status with expression of key cell cycle regulating proteins and survival from colorectal cancer. Diagn Pathol 2013, 8:10.

41. Eberhard J, Gaber A, Wangefjord S, Nodin B, Uhlen M, Ericson Lindquist K, Jirstrom $K$ : A cohort study of the prognostic and treatment predictive value of SATB2 expression in colorectal cancer. Br J Cancer 2012, 106(5):931-938. 
42. Nodin B, Johannesson H, Wangefjord S, O'Connor DP, Lindquist KE, Uhlen M, Jirstrom K, Eberhard J: Molecular correlates and prognostic significance of SATB1 expression in colorectal cancer. Diagn Pathol 2012, 7:115.

43. Katz MH, Hauck WW: Proportional hazards (Cox) regression. J Gen Intern Med 1993, 8(12):702-711.

44. Campbell PT, Jacobs ET, Ulrich CM, Figueiredo JC, Poynter JN, McLaughlin JR, Haile RW, Jacobs EJ, Newcomb PA, Potter JD, et al: Case-control study of overweight, obesity, and colorectal cancer risk, overall and by tumor microsatellite instability status. J Nat/ Cancer Inst 2010, 102(6):391-400.

45. Christodoulides C, Lagathu C, Sethi JK, Vidal-Puig A: Adipogenesis and WNT signalling. Trends Endocrinol Metab 2009, 20(1):16-24.

46. Schinner S: Wnt-signalling and the metabolic syndrome. Horm Metab Res 2009, 41(2):159-163.

47. Morikawa T, Kuchiba A, Yamauchi M, Meyerhardt JA, Shima K, Nosho K, Chan AT, Giovannucci E, Fuchs CS, Ogino S: Association of CTNNB1 (beta-catenin) alterations, body mass index, and physical activity with survival in patients with colorectal cancer. JAMA 2011, 305(16):1685-1694.

48. Maeda K, Chung Y, Kang S, Ogawa M, Onoda N, Nishiguchi Y, Ikehara T, Nakata B, Okuno M, Sowa M: Cyclin D1 overexpression and prognosis in colorectal adenocarcinoma. Oncology 1998, 55(2):145-151.

49. Handa K, Yamakawa M, Takeda H, Kimura S, Takahashi T: Expression of cell cycle markers in colorectal carcinoma: superiority of cyclin A as an indicator of poor prognosis. Int J Cancer 1999, 84(3):225-233.

50. Holland TA, Elder J, McCloud JM, Hall C, Deakin M, Fryer AA, Elder JB, Hoban PR: Subcellular localisation of cyclin D1 protein in colorectal tumours is associated with p21(WAF1/CIP1) expression and correlates with patient survival. Int J Cancer 2001, 95(5):302-306.

51. Bahnassy AA, Zekri AR, El-Houssini S, El-Shehaby AM, Mahmoud MR, Abdallah S, El-Serafi M: Cyclin A and cyclin D1 as significant prognostic markers in colorectal cancer patients. BMC Gastroenterol 2004, 4:22

52. Bondi J, Bukholm G, Nesland JM, Bukholm IR: Expression of non-membranous beta-catenin and gamma-catenin, c-Myc and cyclin D1 in relation to patient outcome in human colon adenocarcinomas. APMIS 2004, 112(1):49-56.

53. Curtin K, Slattery ML, Holubkov R, Edwards S, Holden JA, Samowitz WS: p53 alterations in colon tumors: a comparison of SSCP/sequencing and immunohistochemistry. Appl Immunohistochem Mol Morphol 2004, 12(4):380-386.

54. Zhang ZF, Zeng ZS, Sarkis AS, Klimstra DS, Charytonowicz E, Pollack D, Vena J, Guillem J, Marshall JR, Cordon-Cardo C, et al: Family history of cancer, body weight, and p53 nuclear overexpression in Duke's C colorectal cancer. Br J Cancer 1995, 71(4):888-893.

doi:10.1186/1479-5876-11-293

Cite this article as: Brändstedt et al:: Influence of anthropometric factors on tumour biological characteristics of colorectal cancer in men and women: a cohort study. Journal of Translational Medicine 2013 11:293.

\section{Submit your next manuscript to BioMed Central and take full advantage of:}

- Convenient online submission

- Thorough peer review

- No space constraints or color figure charges

- Immediate publication on acceptance

- Inclusion in PubMed, CAS, Scopus and Google Scholar

- Research which is freely available for redistribution 